\title{
Acute Phase Inflammatory Proteins, Gut Microbiota Dysbiosis and Severity of Nonalcoholic Fatty Liver Disease in Patients with Type 2 Diabetes Mellitus
}

\author{
OANA-ELENA ANCUSA ${ }^{1}$, DOINA GEORGESCU ${ }^{1 *}$, STELA IURCIUC ${ }^{2 *}$, \\ LIVIU-ANDREI GEORGESCU ${ }^{3}$, NORINA BASA ${ }^{1}$, OANA BELEI ${ }^{4}$, DANIEL LIGHEZAN ${ }^{1}$ \\ ${ }^{1}$ Victor Babes University of Medicine and Pharmacy, Department of Internal Medicine, 2 Eftimie Murgu Sq., 300041, \\ Timisoara, Romania \\ ${ }^{2}$ Victor Babes University of Medicine and Pharmacy, Department of Cardiology, 2 Eftimie Murgu Sq, 300041, Timisoara, \\ Romania \\ ${ }^{3}$ Victor Babes University of Medicine and Pharmacy, Department of Nephrology, 2 Eftimie Murgu Sq., 300041, Timisoara, \\ Romania \\ ${ }^{4}$ Victor Babes University of Medicine and Pharmacy, Department of Pediatrics, 2 Eftimie Murgu Sq., 300041, Timisoara, \\ Romania
}

\begin{abstract}
Since obesity and type 2 diabetes mellitus (DM) became almost pandemic, incidence and prevalence of associated nonalcoholic fatty liver disease (NAFLD) rose accordingly. Aim of the study was assessing whether there is a link between circulating $C$-reactive protein(CRP), gut microbiota dysbiosis and severity of liver disease in patients with NAFLD and confirmed type 2 DM. 50 patients diagnosed with type 2 DM were consecutively enrolled in this cross-sectional, pilot study, being evenly divided in two groups, as matched pairs, on the basis of presence or absence of NAFLD confirmed by abdominal and CT exam, after ruling out a lot of diseases and conditions. Patients underwent measurements of waist circumference, blood pressure (BP), body mass index (BMI) and thoroughly physical examination. Complete blood count (CBC), liver tests, CRP, fasting plasma glucose (FPG), low density lipoprotein $(L D L)$ and high-density lipoprotein $(H D L)$ cholesterol, triglycerides, creatinin and uric acid, micro proteinuria as well as stool microbiology were run, using standardized methods. Fatty liver index (FLI) was also calculated. Fibromax (BioPredictive) was performed in NAFLD (+) patients. NAFLD (+) group displayed significant differences of age and BMI, as well as lab biochemistry: ALT, GGT, LDL-cholesterol and triglycerides, CRP, microprotienuria and stool microbiology. Strong positive correlation of CRP with gut dysbiosis (DB) severity, microproteinuria, as well as with nonalcoholic steatohepatitis (NASH) score, were observed. DB range correlated positively in NAFLD (+) group with age, BMI, BP, dyspepsia as well as with NASH scores. In conclusion patients with NAFLD and associated type 2 DM displayed a specific profile with significant differences of age, BMI, FLI, diabetic treatment, biochemistry and gut microbiological charts. Strong positive correlations between CRP, gut DB and NASH score suggest the possibility of link between inflammation, gut microbiota $D B$ and severity of liver disease.
\end{abstract}

Keywords: CRP, gut microbiota dysbiosis, NAFLD, type 2 DM

\section{Introduction}

Nonalcoholic fatty liver disease (NAFLD) is a complex entity characterized by $\geq 5 \%$ accumulation of fat in liver cells, in the absence of excessive alcohol consumption or any other secondary causes, ranging from simple steatosis to inflammation and hepatocytes necrosis known as NASH (nonalcoholic steatohepatitis), or end stage liver disease (NASH-induced cirrhosis): fibrosis and architectural liver changes $[1,2]$. Since obesity and type 2 DM became almost pandemic, incidence and prevalence of NAFLD rose accordingly. It is estimated that in Asia and USA, between 2005 and 2010, the prevalence of NAFLD increased exponentially [3].

\footnotetext{
$\overline{\text { *emaildoina.georgescu@umft.ro,siurciuc@umft.ro }}$
} 
The real burden of NAFLD remains yet obscure, since the diagnosis is probably highly underrated and not always accurate, based mostly on imagistic approaches and seldom as a result of liver biopsy, not to mention numerous associated health conditions that multiply its morbidity and mortality. Since NAFLD rarely has characteristic symptoms or signs, diagnostic is frequently incidental. Two situations drive the clinical diagnostic: either increase of transaminases and GGT or imagistic features consistent with liver steatosis. Given the liver biopsy is not systematically used as a diagnostic method, noninvasively approach is lately preferred, especially for screening NAFLD. Liver ultrasound, CT or MRI are currently accepted as reliable methods to detect fatty infiltration. However, there is a difference of diagnostic sensitivity, when it comes to method, which consecutively reflects in large variation of epidemiological data reporting NAFLD. For several reasons ultrasonography is considered the method of choice for screening medium to severe fatty liver. [4-6] If diagnosis is established in end stage liver disease, patients will display, as all patients with liver cirrhosis, no matter etiology, liver stigmata signs along with portal hypertension and severe alterations of biochemical liver tests.

So called primary NAFLD, often associated with central obesity, dyslipidemia, insulin resistance and diabetes mellitus, became nowadays more and more serious. That results in important problems of public health, especially in developed countries, not only related to its increasing incidence, but also due to its potential of evolution toward severe hepatic lesions including liver fibrosis, cirrhosis and its complications, such as portal vein thrombosis, and hepatocellular carcinoma [7,8].

As many studies recently reported, various factors consistent with "multiple hit theory" could at some point trigger liver injury, resulting in inflammation and hepatocytes necrosis and consecutively could promote liver fibrosis. These factors could be genetic, insulin resistance, dietary habits with high carbohydrates and fats, hormones imbalance and, gut microbiota dysbiosis (DB).[9] Gut microbiota DB seems to play an important part in NAFLD pathogenesis and could intervene in modulation the energy metabolism, resulting in fat accumulation as droplets of triglycerides, as well as in development of insulin resistance, increase of FFA production, as well as decrease of choline production and increase of many hepatotoxic compounds [10].

Aim of the study was assessing whether there is a link between inflammation, gut microbiota DB and severity of liver disease in patients with NAFLD and type 2 DM.

\section{Patients and methods}

\subsection{Study design and methodology}

2.1.1Inclusion criteria: 50 patients diagnosed with type 2 diabetes mellitus were consecutively enrolled in this cross-sectional, pilot study, being evenly divided in two groups, as matched pairs, on the basis of presence or absence of NAFLD confirmed by abdominal ultrasonography and CT exam. Study was approved by the Ethics Committee of Scientific Research of the University of Medicine and Pharmacy "Victor Babes" from Timisoara, Romania and was conducted in accordance with the Declaration of Helsinki. All participants provided written informed consent before the beginning of the study.

2.1.2 Exclusion criteria: heavy consumption of alcohol: more than $40 \mathrm{gr} /$ day in men and more than $30 \mathrm{gr} /$ day in women, in past 10 years, significant exposure to industrial toxic substances or to medication with liver toxicity, hemochromatosis, alpha 1 antitripsine deficiency, Wilson disease, autoimmune hepatitis, infection with viral $\mathrm{B}$ or viral $\mathrm{C}$ hepatitis, thyroid diseases, various organ failure (cardio-pulmonary, kidney or liver), oncologic conditions, long parenteral nutrition, associated inflammatory bowel disease, celiac disease or gluten sensitivity, various infectious diseases, malnutrition syndromes, as well as recent treatment with antibiotics or probiotics.

2.2 Protocol of examination and laboratory work-up Patients underwent measurements of waist circumference and blood pressure (BP), BMI assessment, as well as thoroughly clinical examination. Laboratory work up: complete blood count $(\mathrm{CBC})$, routine liver tests including HBs antigen, Delta 
antigen, anti $\mathrm{HCV}$ antibodies, plasma iron and copper, alpha 1 antitripsine, antinuclear antibodies (ANA), antimitochondrial antibodies (AMA), as well as high sensitive C-reactive protein (CRP), fasting plasma glucose (FPG), cholesterol low-density lipoprotein(LDL) and high-density lipoprotein(HDL), triglycerides, creatinin and uric acid, microproteinuria, urine and stool microbiology were run, using standardized methods After. identifying different types of stool species by matrixassisted laser desorbtion ionization-time of flight -mass spectrometry( MALDI-TOF-MS) method, the severity of gut microbiota DB, i, was semiquantitative scored as: $0=$ absent, $1=$ mild, $2=$ medium, $3=$ severe [11].

2.3 C-reactive protein (CRP) is a nonglycosilated plasma protein, with an annular shape and pentameric structure, consisting of five conformational protein subunits, with high resistance to proteolysis, that migrates near the gamma zone on electrophoresis. It is synthesized by many immune cells with various locations, predominantly in the liver, as a reaction of proinflammatory interleukins and cytokines. Occasionally could be produced de novo in extrahepatic sites. Because its levels rise and fall depending on the amount of inflammation or tissue lesions in the body, CRP is also known as an acute phase reactant. To determine CRP, venous blood samples were collected in the morning, in fasting patients, within vacutainers and processed after centrifugation, using immunoturbidimetry assay. The cutoffs were $0.5 \mathrm{mg} / \mathrm{dL}$ [12].

\subsection{Non invasive assessment of NAFLD}

2.4.1. Abdominal helical $\mathrm{CT}$, as well as real-time high resolution ultrasonography were used to confirm or rule out fatty liver disease in study participants. Lipid accumulation determines decrease of liver density, which becomes less dense when compared to spleen. It is considered that a decrease more than 10 Hounsfield units (HU) on a native CT is consistent with diagnosis of liver steatosis, while using contrast the difference between liver and spleen augments to more than $20 \mathrm{HU}$. Abdominal ultrasonography was performed in all study participants, with semiquantitative assessment of steatosis, when observed, as follows: mild, moderate and severe [13].

2.4.2. Fibromax, developed by BioPredictive $\AA$, consistent with 5 different noninvasive tests, from which: FibroTest ${ }^{\circledR}$, SteatoTest ${ }^{\circledR}$ and NashTest ${ }^{\circledR}$ were actually used in this study, in the evaluation of NAFLD (+) group. This complex test is based on validated mathematical application, that uses some biochemical blood variables, such as: alpha-2 macroglobulin, haptoglobin, apolipoprotein A1, total bilirubin, gamaglutamiltranspeptidase ( GGT), alanin - aminotransferase (ALT), aspartat aminotransferase (AST), fasting plasma glucose (FPG), cholesterol and triglycerides, as well as some clinical data such as: age, gender, weight and height, in order to determine the severity of liver disease [14].

2.4.3. Fatty liver index (FLI), as instrument of prediction of fatty liver risk was calculated using a mathematical formula based on measurements of waist circumference $(\mathrm{cm}), \mathrm{BMI}\left(\mathrm{kg} / \mathrm{m}^{2}\right)$, level of GGT (U/l) and triglycerides (mg/dl).The FLI formula that calculates the likelihood of fatty liver disease was: $\mathrm{FLI}=(\mathrm{e} 0.953 * \log$ e (triglycerides $)+0.139 * \mathrm{BMI}+0.718 * \log$ e $(\mathrm{GGT})+0.053 *$ waist circumference -15.745$) /(1+$ e $0.953 * \log$ e (triglycerides $)+0.139 * \mathrm{BMI}+0.718 * \log$ e $(\mathrm{GGT})+$ $0.053 *$ waist circumference - 15.745) x 100 . The values range between 0 and 100 . A FLI $<30$, basically rules out a fatty liver (FL), whereas a FLI>60 represents a high risk for FL and mandates further exams [15].

2.5 Assessment of DM According to American Diabetes Association (ADA) criteria, a FPG of 126 $\mathrm{mg} \%$ or higher, or a 2 hour plasma glucose level of $200 \mathrm{mg} \%$ during $75 \mathrm{~g}$ oral glucose tolerance test, are consistent with the diagnosis of DM [16]. 
2.6 Statistical analysis Graph Pad Prism 8.3.1 software (Graph Pad Software, Inc., La Jolla, CA, USA) was used for statistical analysis. Given exploratory, pilot study, no sample size calculation was needed. Quantitative variables were expressed as mean values (MV) \pm standard deviation (SD). Chisquared test was used to compare the two of groups, in cases of qualitative variables expressed as percentages. The unpaired t test was calculated, $\mathrm{p} \leq 0.05$ was considered statistically significant, with confidence interval $\mathrm{CI}=95 \%$.Nonparametric Pearson's correlation test was also performed in order to establish the $\mathrm{r}$ coefficient, drawing the direction and magnitude of possible links between variables.

\section{Results and discussions}

As depicted in Table 1, analysis of demographical and clinical characteristics in study participants showed significant statistically differences related to age $(\mathrm{p}=0.0294)$, BMI $(\mathrm{p}=0.0084)$, FLI $(\mathrm{p}=0.0001)$ and therapy of DMThus, NAFLD $(+)$ patients needed insulin in significantly higher percentage to compensate DM ( $\mathrm{p}<0.0001)$, while those without NAFLD were treated mostly with oral antidiabetics and no insulin $(\mathrm{p}<0.0001)$.In respect to treatment of dyslipidemia, significant differences related to statins were seen in favor of NAFLD positive group $(\mathrm{p}=0.0035)$.

Table 1. Baseline demographical and clinical characteristics in study participants

\begin{tabular}{|l|l|l|l|}
\hline & NAFLD(+) & NAFLD(-) & $\mathrm{p}$ \\
\hline Age(years)* & $72.28 \pm 8.88$ & $66.32 \pm 9.86$ & $\mathbf{0 . 0 2 9 4}$ \\
\hline Gender females/males & $8 / 17$ & $13 / 12$ & 0.1561 \\
\hline Residence urban/rural & $15 / 10$ & $13 / 12$ & 0.5727 \\
\hline BMI(kg/m $)^{*}$ & $31.03 \pm 7.37$ & $26.6 \pm 3.48$ & $\mathbf{0 . 0 0 8 4}$ \\
\hline SBP(mmHg) & $143.00 \pm 27.16$ & $144.00 \pm 26.18$ & 0.8951 \\
\hline fatigue & $40 \%$ & $32 \%$ & 0.5597 \\
\hline dyspepsia & $48 \%$ & $32 \%$ & 0.2530 \\
\hline FLI (units)* & $77.52 \pm 10.56$ & $32.08 \pm 14.61$ & $\mathbf{0 . 0 0 0 1}$ \\
\hline Cardio-vascular conditions & $100 \%$ & $100 \%$ & 1 \\
\hline Treatment -only diet & $0 \%$ & $8 \%$ & 0.1538 \\
\hline \multicolumn{1}{|c|}{ Oral antidiabetics* } & $36 \%$ & $92 \%$ & $<\mathbf{0 . 0 0 0 1}$ \\
\hline Insulin therapy* & $64 \%$ & $0 \%$ & $<\mathbf{0 . 0 0 0 1}$ \\
\hline Statins* & $84 \%$ & $44 \%$ & $\mathbf{0 . 0 0 3 5}$ \\
\hline Statins +fibrates & $16 \%$ & $12 \%$ & 0.6866 \\
\hline
\end{tabular}

legend: $\mathrm{BMI}=$ body mass index, $\mathrm{SBP}=$ systolic blood pressure, $\mathrm{FLI}=$ fatty liver index,*; bold =statistically significant

As illustrated in Table 1 and Figure 1, FLI calculated in all study participants, showed in NAFLD (+) group a highly statistically significant difference (77.52 U \pm 10.56 vs. $32.08 \mathrm{U} \pm 14.61 ; \mathrm{p}=0.0001)$.

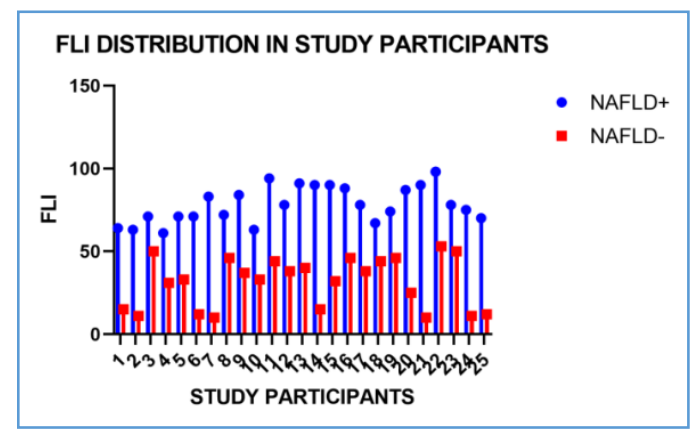

Figure 1. FLI distribution: NAFLD (+) vs. NAFLD (-) participants

Analysis of patients biological charts revealed statistically significant differences in favor of first group, respectively NAFLD $(+)$, regarding transaminases-ALT $(p=0.023)$, GGT $(p=0.0064)$, plasma 
lipids: LDL $(\mathrm{P}=0.0309)$ and triglycerides $(\mathrm{p}=0.0506)$, CRP level $(\mathrm{p}=0.0416)$, as well as microproteinuria $(\mathrm{p}=0.0007)$ and $\mathrm{DB}$ range $(\mathrm{p}<0.0001)$. These data are displayed in Table 2.

Table 2. Biological characteristics in study participants

\begin{tabular}{|c|c|c|c|}
\hline & NAFLD $(+)$ & NAFLD(-) & $\mathrm{p}$ \\
\hline $\mathrm{Hb}(/ \mathrm{dl})$ & $12.74 \pm 1.73$ & $12.29 \pm 2.23$ & 0.4288 \\
\hline $\mathrm{L} / \mathrm{mm}^{3}$ & $8.68 \times 10^{3} \pm 2.10 \times 10^{3}$ & $9.58 \times 10^{3} \pm 2.79 \times 10^{3}$ & 0.2049 \\
\hline $\mathrm{Plt} / \mathrm{mm}^{3}$ & $238.04 \times 10^{3} \pm 68.3 \times 10^{3}$ & $225.36 \times 10^{3} \pm 90.86 \times 10^{3}$ & 0.5795 \\
\hline $\mathrm{ALT}(\mathrm{iu} / \mathrm{l}){ }^{*}$ & $45.2 \pm 40.82$ & $25.16 \pm 12.4$ & $\mathbf{0 . 0 2 3}$ \\
\hline $\mathrm{GGT}(\mathrm{u} / \mathrm{l})^{*}$ & $51.52 \pm 33.2$ & $31.56 \pm 11.07$ & $\mathbf{0 . 0 0 6 4}$ \\
\hline LDL cholesterol(mg/dl) $*$ & $107.2 \pm 35.81$ & $86.8 \pm 28.64$ & $\mathbf{0 . 0 3 0 9}$ \\
\hline HDL coletserol(mg/dl) & $41.56 \pm 13.03$ & $40.68 \pm 8.42$ & 0.7779 \\
\hline Triglycerides(mg/dl) $*$ & $184.96 \pm 166.01$ & $119.76 \pm 31.31$ & $\mathbf{0 . 0 5 0 6}$ \\
\hline Creatinin(mg/dl) & $1.44 \pm 0.79$ & $1.24 \pm 0.56$ & 0.3113 \\
\hline FPG(mg/dl) & $171.96 \pm 50.62$ & $142.96 \pm 44.93$ & 0.0373 \\
\hline Uric acid(mg/dl) & $6.75 \pm 1.78$ & $6.8 \pm 1.65$ & 0.9220 \\
\hline CRP(mg/dl) $*$ & $21.47 \pm 27.17$ & $9.7 \pm 7.0$ & $\mathbf{0 . 0 4 1 6}$ \\
\hline Microproteinuria* & $80 \%$ & $32 \%$ & $\mathbf{0 . 0 0 0 7}$ \\
\hline Gut DB* & $88 \%$ & $28 \%$ & $<\mathbf{0 . 0 0 0 1}$ \\
\hline
\end{tabular}

Legend: $\mathrm{Hb}=$ hemoglobin, $\mathrm{L}=$ leukocytes, $\mathrm{Plt}=$ platelets, $\mathrm{FPG}=$ fasting plasma glucose,

$\mathrm{CRP}=\mathrm{C}$ reactive protein, $\mathrm{DB}=$ dysbiosis,,$*$; bold $=$ statistically significant

As depicted in Figure 2A and 2B, CRP displayed strong positive correlation with gut DB ( $\mathrm{r}=0.67$, $\mathrm{p}=0.0092)$ and NASH score $(\mathrm{r}=0.51, \mathrm{p}=0.008)$.


Figure 2. CRP correlations in NAFLD (+) group: 2A to gut DB, 2B. to Fibromax scores

Correlation studies applied to DB illustrated a strong link with different demographical and clinical data, as follows: age $(\mathrm{r}=0.38, \mathrm{p}=0.05), \mathrm{BMI}(\mathrm{r}=0.63$, $\mathrm{p}-9.996), \mathrm{SBP}(\mathrm{r}=0.59, \mathrm{p}=0.0019)$ and dyspepsia $(\mathrm{r}=0.63, \mathrm{p}=0.0002)$, as seen in Figure $3 \mathrm{~A}$.

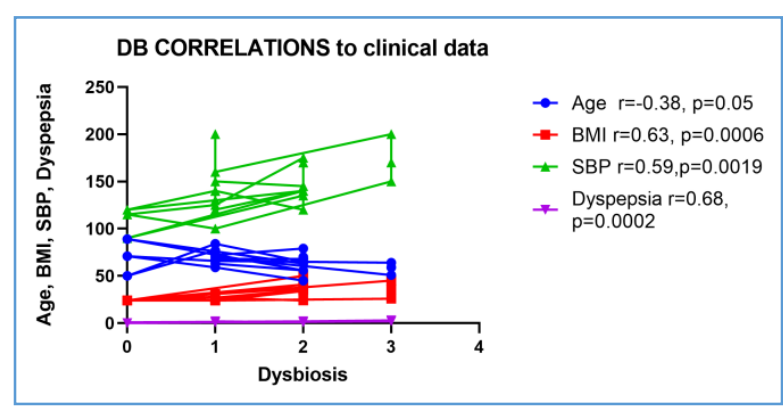

Figure 3A. Correlations of gut DB to demographical and clinical data in NAFLD (+) group 
Related to Fibromax scores, DB positively good correlated to NASH score, respectively: $r=0.46$, $\mathrm{p}=0.01$. These aspects are depicted in Figure 3B.

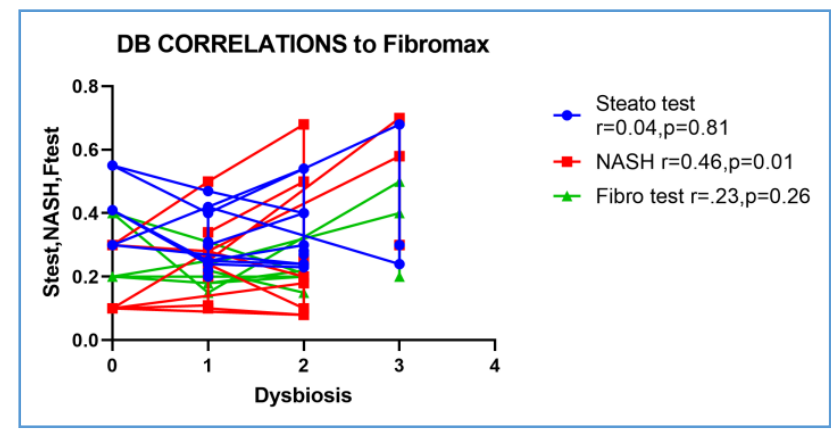

Figure 3B. Correlations of gut DB to

Fibromax scores in NAFLD $(+)$ group

As seen in Figure 3C, DB also strong positively correlated to microproteinuria; $r=0.58, \mathrm{p}=0.019$.

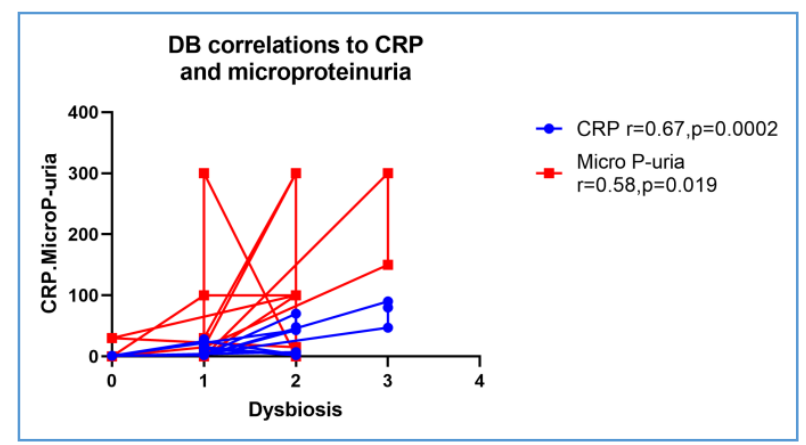

Figure 3C. Correlations of gut DB to CRP and microproteinuria in NAFLD (+) group

In accordance with others, we observed that NAFLD+ group displayed significant older age by comparison to NAFLD- participants. It seems that incidence and prevalence of NAFLD increase with growing old, also resulting in more severe biochemical and histological features [17].

As others reported, we also observed that FLI showed utility and reliability in the assessment of risk for liver steatosis, while all study participants with confirmed NAFLD exhibited levels above 60 units [18].

Whether current, DZ treatment influenced results of this study, in respect to presence and severity of fatty liver, it is however not clear. Patients with the second group (NAFLD negative), were mainly treated with oral antidiabetic drugs (OAD) (metformin), while the group displaying NAFLD did not further receive $\mathrm{OAD}$, patients becoming insulin necessitant. In this regard many multicenter studies and meta-analysis investigated relation between various OAD and NAFLD, but results are neither coherent nor homogeneous, and rather contradictory. However, to date, European Association for Study of the Liver (EASL) did not yet introduce any specific recommendation of drugs in the guidelines for NAFLD treatment [19]. To record, all patients in this study with NAFLD received lipid lowering agents, basically statins, in a significant higher perecentage, by comparison to those without NAFLD and a few of them combination of statins with fibrates. Even NAFLD group was basically treated with antidiabetics and statins, they showed elevated transaminases amd GGT level. However, there is yet no conclusive evidence that dyslipidemia treatment with any kind of agent either statins, fibrates, fish oil, etc,, could significantly improve liver histology severity or outcome in NAFLD patients [20]. 
Gut microbiota DB, obesity and metabolic syndrome are close related, as confirmed by many recent studies, including ours, conducted both in animal and humans, by modulation of host nutritional and energy metabolic pathways. No less important seems to be so called metabolic endotoxinemia, as a part of this complex process, related to overgrowth of some microbial gut species and reduction of local flora biodiversity, with increased ratio Firmicutes to Bacteroidetes. These aspects correlate with adiposity, insulin resistance and proinflammatory status.[21] Interestingly, metformin seems to stimulate bacteria species that produce butyrate and consecutively promote normobiosis or a healthy microbiome.[22] In accordance with these observations we have recorded significant lower rates of dysbiosis severity in patients without NAFLD, treated by OAD-metformin.

Inflammation, gut DB and NAFLD seem to be linked, as many studies support this hypothesis. The bioenergetic pathways related to mithocondria activity, immunometabolism with pivotal, biochemical reactions, performed by numerous immune cells effectors, could be reprogrammed under particular stimuli. One of these stimuli that can drive host immunological functions is represented by gut mibrobiota DB. DB could definitively affect the ability of adaptative, inflammatory responses and trigger proinflammatory cells with release of various interleukins and cytokines, eventually altering the host metabolism.[23] In this regard, we have noticed a positively strong correlation not only between the levels of CRP and range of DB severity, as well as NASH scores, but also directly between DB severity itself and NASH scores.

Results of the present study have also highlighted strong positive correlations between range of DB and several clinical data, such as: age, BMI and SBP. Many recent studies advocated the role of $\mathrm{DB}$ in the setting of cardio-vascular risk, via trimethylamine $\mathrm{N}$-oxide, short chain fatty acids and secondary bile acids, that interfere various signaling and communication pathways, resulting in hypertension, atherosclerosis and coronary artery disease. Otherwise cardio-vascular comorbidities represent another important issue to manage in NAFLD population, as we also observed in present study [24].

There is an increased risk of associated microproteinuria and chronic kidney disease (CKD) in patients with NAFLD, as a recently meta-analysis concluded. [25] Our study identified significant increased incidence of microalbuminuria in NAFLD (+) group, suggesting the importance of screening for CKD in population already affected by NAFLD, not to mention the risk for cardio-vascular diseases. We also observed a strong correlation between microalbuminuria levels and DB severity.

Given data from present study and many others, inflammation is a fundamental process, implicated in many conditions, orchestrated by gut microbiota DB, that triggers other participants either local or distant, which further aggravates the initial condition.[26] Since the understanding of this basic process associates DB with inflammation, a lot of scientists embraced the idea of natural remedies, such as probiotics, in order to influence this pathophysiological pathway and the occurrence or development of many disorders. [27] In this regard, other potential natural antiinflammatory formulas could be taken into account, based on their promising preliminary results [28,29]. No less important will be to improve our possibility to predict the risk for DM and its complications, including NAFLD. [30]

Limitations This observational study has a few limitations. Even if it is a pilot study, though the sample size of study population is relatively small, with consecutive potential statistical draw-backs. Another limitation is related to methodology of NAFLD diagnostic. Although the diagnosis protocol was homogenous, it did include only noninvasive methods, which could trigger ambiguity, considering that liver biopsy remains the gold standard in liver diseases diagnosis. Not least, given the crosssectional study, a lot of statistical bias could also intervene, related to sources of information bias and possible confounders.

\section{Conclusions}

Patients with type 2 DM that developed NAFLD displayed a profile characterized by older age, higher BMI and FLI range, necessity of insulin therapy, modified biological charts that included acute inflammatory phase protein- CRP, liver tests, serum circulating lipids, microproteinuria, as well as 
higher range of gut DB. Strong positive correlations observed between CRP, gut DB and NASH and also between DB, BMI and NASH suggest the possibility of link between inflammation, gut microbiota DB and severity of liver disease.

\section{References}

1.WILLIAMS CD, STENGEL J, ASIKE MI et al. Prevalence of NAFLD and NASH among a largely middle -aged population utilizing ultrasound and liver biopsy: a prospective study. Gastroenterology 140, 2011, p.124

2.CHASALANI N, YOUNOSSI Z, LANVINE JE et al. The diagnostic and management of NAFLD: Practice guidance from AASLD. Hepatology 67, 2018, p.328

3.PERUMPAIL BJ,KHAN MA,YOO ER et al. Clinical epidemiology and disease burden of NAFLD World J Gastroenterol 23, no.47,2017, p.8263

4.GEORGESCU D, COCEALA L, BASA $\mathrm{N}$ et al. Ultrasound in the assessment of fatty liver in patients with metabolic syndrome Ultraschall in der Medizin. S29, 2008, S6.

5.HERNAEZ R, LAZO R, BONEKAMP $S$ et al. Diagnostic accuracy and reliability of ultrasonography for the detection of fatty liver: a meta-analysis. Hepatology 54, 2011, p.1082

6.SIMA A, SPOREA I, TIMAR R et al. Noninvasive assessment of liver steatosis and fibrosis using TE and controlled attenuation parameter in type 2 diabetes patients. Acta Endocrinologica, 14, no.3, 2018,p.394

7.STINE JG, SHAH NL, ARGO CK, et al. Increased risk of portal vein thrombosis in patients with cirrhosis due to nonalcoholic steatohepatitis. Liver Transpl. 21,2015,p.1016

8.GEORGESCU D, IURCIUC M, IONITA I et al. Portal vein thrombosis and gut microbiota: understanding the burdem. Rev. Chim., 70, (6), 2019, 2181

9.BUZZETTI E, PINZANI M,TSOCHATZIS EA The multiple-hit pathogenesis of NAFLD Metabolism. 65,2016,p.1038

10.LEUNG C, RIVERA L, FURNESS JB,ANGUS PW. The role of gut microbiota in NAFLD.Nat Rev Gastroenterol and Hepatol. 13, no.7, 2016, p.412

11.SANDRIN TR, GOLDSTEIN JE and SHOEMAKER S. MALDI TOF MS profiling of bacteria at the strain level: A review. Mass Spectrometry Reviews 32, no.3, 2013, p.188

12.WU I, POTEMPA LA, EL KEBIR D, FILEP JG. C-reactive protein and inflammation, Biol Chem 396, 2015, p.1181

13.LI Q, DHYANI M, GRAJO JR, et al. Current status of imaging in NAFLD. World J Hepatol 10, no.8, 2018, p.530

14.MORRA R, MUNTEANU M, IMBERT-BISMUT $F$ et al. Fibromax: towards a new universal biomarker of liver disease? Expert Rev Mol Diagn 7, 2007, p.5

15.BEGOGNI G, BELLENTANI S, MIGLIOLI L et al. The fatty liver index: a simple and accurate prediction of hepatic steatosis in general population. BMJ Gastroenterol. 6, no.1, 2006, p.33

16.***Diagnostic and classification of diabetes mellitus. American Diabetes Association .Diabetes Care 33, suppl1, 2010, p.32

17.FRITH J, DAY CP, HENDERSON E, BURT AD, NEWTON JL. NAFLD in older people.Gerontology 55, 2009, p.607

18. KOEHLER EM, SCHOUTEN JN, HANSEN BE et al. External validation of the fatty liver index for identifying NAFLD in a population-based study. Clin Gastroenterol Hepatol 11, 2013, p.1201

19. KOTHARI S, DHAMI-SHAH H, SHAH SR ,Antidiabetic drugs and statins in NAFLD.J Clin Experim Hepatol. 9,no. 6,2019,p.723

20.ZHANG QQ, LU LG.Nonalcoholic fatty liver disease: dyslipidemia, risk for cardio-vascular complications and treatment strategy. J Clin Transl Hepatol.3, no.1, 2015, p.78

20.FESTI D, SCHIUMRINI R, EUSEBI LH et al. Gut microbiota and metabolic syndrome World J Gastroenterol 20, no.43, 2014, p.16079 
21.FORSLUND K, HILDEBRAND F, NIELSEN T et al. Disentangling type 2 diabetes and metformin treatment signatures in the human gut microbiota.Nature. 528, no.7581, 2015, p.262

22.SALZMAN ET,PALACIOS T,THOMSEN M,VITETTA L.Intestinal microbiome shifts, dysbiosis, inflammation and nonalcoholic fatty liver disease.Front Microbiol 9, 2018, p.61

23.JIN M, QIAN Z,YIN J,XU V,ZHOU X.J. The role of intestinal microbiota in cardio-vascular disease. Cell Mol Med. 23. No.4, 2019, p.2343

24.WIJAMPREECHA K, THONGPRAYOON C, BOONPHENG B et al. NAFLD and albuminuria: a systematic review and meta-analysis. Eur J Gastroenterol Hepatol. 30, no.9, 2018,p.986

25.BELIZARIO JE, FAINTUCH J, GARAY-MALPARTIDA M. Gut microbiome dysbiosis and immunometabolism: new frontiers for treatment of metabolic diseases. Hindawi. Mediators of Inflammation. 2018, p.2037838

26.REID G, YOUNES JA, VAN DER MEI HC et al. Microbiota restoration: natural and supplemented recovery of human microbial communities. Nat Rev Microbiol. 9, no.1,2011, p.27

28.ISAIA IA, IENASCU IMC, ANDRICA FM et al. Preliminary in vitro evaluation of seven different plant extracts on A375, B1664A5 and HaCat cell lines. Rev. Chim., 67, (8), 2016, 1633

29.NICOLOV M, DUSE AO, GEORGESCU D et al. Preliminary studies on betulinic acid crystals with organic solvents. Rev. Chim., 87, (7), 2016, 1411

30.JURCA-SIMINA IE, JUGANARU I, IURCIUC MS et al. What if body fat percentage association with FINDRISC score leads to a better prediction of type 2 diabetes mellitus?Rom J Morphol Embryol 60, no.1, 2019, p.205

Manuscript received: 20.02 .2020 